combined 18FDG PET/MRI imaging system in an AMI mouse model with the aim of acquiring simultaneous morphological and functional data.

Methods An open-chest mouse (C57Bl/6J) heart model was utilised in which the left anterior descending coronary artery was occluded for 30 minutes to induce an ischaemic insult. A cardiac MRI with a 4.7T Bruker BioSpec system was performed $24 \mathrm{~h}$ after surgery. At 4 weeks post-AMI a second MRI was performed followed by a combined 18FDG PET-MRI investigation using the Cambridge split-magnet system.

Results Gd-enhanced MRI at 24 hr reperfusion revealed hyperenhancement of the infarcted region which corresponded to a signal void in the PET image obtained at 4 weeks post-MI. Furthermore, this was associated with a decrease in global ejection fraction as well as left ventricular wall thinning, consistent with the onset of heart failure.

Conclusion These preliminary findings demonstrate that the combined PET/MRI imaging system is a powerful tool in studying the pathophysiology of CHF allowing a better understanding of the progressive changes that occur and the direct evaluation of novel therapeutic treatments.

\section{AN MRI COIL DUAL-TUNED BY RF SHIELDING FOR IN-VIVO DETECTION OF FLUORINE LABELLED STEM-CELLS IN A RODENT MODEL OF ACUTE MYOCARDIAL INFARCTION}

\section{Presenter: Lowri Cochlin}

doi:10.1136/heartjnl-2012-302951.025

'LE Cochlin, 1,2,3RSM Gomes 'K Clarke, 'CA Carr. 'Cardiac Metabolism Research Group, Department of Physiology, Anatomy and Genetics, University of Oxford, UK; 2Biomaterials and Stem-Cells Based Therapeutics Lab, Biocant, Cantanhede, Portugal; ${ }^{3}$ Centre of Neurosciences and Cell Biology, University of Coimbra, Portugal
Stem-cell therapy holds great promise for treatment of disease. However, optimal stem-cell type, number, route, timing of administration have yet to be quantitatively determined. Non-invasive methods of monitoring cell retention are therefore in demand. Fluorine-MRI offers the facility for unambiguous localisation of fluorine-labelled cells as they migrate in-vivo. We achieve high fluorine sensitivity by simple modification to a standard MRI-coil. We monitored stem-cell retention in a rat model of myocardial infarction (MI), for 13 days after administration of fluorine-labelled stem-cells

Methods MRI-coil: $65 \mathrm{~mm}$ diameter, low-pass birdcage fitted with two RF-shields; $85 \mathrm{~mm}$ removable, $79 \mathrm{~mm}$ fixed. Inner shield removal causes the resonant frequency window to shift from proton to fluorine. Labelled cell preparation and administration: cardiac-derived stem-cells were incubated with polylactic-co-glycolic acid (PLGA) nanoparticles containing perfluoro(crown)ether (PFCE) and fluoresceinamine. Five million labelled cells were administered immediately post MI, induced by occlusion of the left descending coronary artery. MRI at 1, 5, 7 and 13 days post MI, using a 7 Tesla magnet. Cardiacgated gradient-echo cardiac long-axis and short-axis stacks: 1 proton average (Texp 25s), 15 fluorine averages, (Texp 380s) TE $=2.9 \mathrm{~ms}$, $\mathrm{FA}=$ Ernst angle $(\mathrm{TR}=\mathrm{R}$ Rinterval $\sim 200 \mathrm{~ms}, \mathrm{~T} 1(\mathrm{PFCE})=1090 \pm 15 \mathrm{~ms}$ in vivo), FOV $=50 \times 50 \times 1.5 \mathrm{~mm}$, matrix $=128 \times 128$.

Results In-vivo, fluorine signal was easily located for setup, and high-resolution fluorine images showed signal co-registered with infarction and operation wound. At day 13, hearts were excised for histological verification of MRI findings.

Conclusion We demonstrate high proton/fluorine sensitivity using a standard MRI-coil, modified for inductance tuning to fluorine. Labelled cardiac stem-cells were imaged for 13 days post MI. Whole-heart proton and fluorine image stacks of matched resolution were acquired within one-hour scan-time. 